\title{
La profesionalización de la función pública local en América Latina: evolución, modelos y propuestas $^{1}$
}

\author{
Federico Amador Castillo Blanco \\ Profesor Titular de Derecho Administrativo. Universidad de Granada \\ Javier Eduardo Quesada Lumbreras \\ Becario de Investigación de la Unión Iberoamericana de Municipalistas
}

\begin{abstract}
Sumario: I. INTRODUCCIÓN: LA SITUACIÓN DE LOS SISTEMAS DE SERVICIO CIVIL O FUNCIÓN PÚBLICA LOCAL EN AMÉRICA LATINA. 1. Evolución de las reformas de modernización del Estado implementadas hasta la actualidad. 2. Los distintos modelos de servicio civil existentes en el ámbito comparado: sistemas abiertos vs. sistemas cerrados. 3. Balance de las experiencias internacionales: luces y sombras del impacto de la globalización de la gestión pública. II. PROPUESTAS PARA LA CONSTRUCCIÓN DE UNA FUNCIÓN PÚBLICA LOCAL DE BASE PROFESIONAL EN AMÉRICA LATINA. 1. Hacia la profesionalización de los servidores públicos en el ámbito local: variables de carácter esencial e instrumental. 2. Los desafíos pendientes para alcanzar el éxito de las reformas emprendidas.
\end{abstract}

\section{INTRODUCCIÓN: LA SITUACIÓN DE LOS SISTEMAS DE SERVICIO CIVIL O FUNCIÓN PÚBLICA LOCAL EN AMÉRICA LATINA}

\section{Evolución de las reformas de modernización del Estado implementadas hasta la actualidad}

A lo largo del siglo XX se han producido numerosas iniciativas orientadas a modernizar los aparatos públicos de buena parte de los distintos países latinoamericanos; aunque con una gran variabilidad, debido en gran medida a las trayectorias diversas de sus sistemas socio-políticos, la mayoría de los países se encuentran inmersos en un importante proceso de reforma de sus respectivos Estados.

En estas iniciativas modernizadoras pueden diferenciarse hasta tres fases. Una primera fase cubre las iniciativas modernizadoras del Estado que surgieron a principios del siglo xx y se prolongaron hasta los años ochenta. Aunque con diferencias en cuanto al punto de partida, la mayor parte de los países de la región iniciaron reformas encaminadas a reformar y reforzar los apa-

\footnotetext{
${ }^{1}$ El presente artículo se realiza en el marco de una colaboración con la Fundación Internacional y para Iberoamérica de Administración y Políticas Públicas (FIIAPP), como parte de un trabajo de análisis aplicado sobre el tema. Asimismo, se enmarca en una de las líneas de investigación del Proyecto de Excelencia «Fortalecimiento institucional de los municipios para la lucha contra la exclusión social y la pobreza en América Latina» que cuenta con la financiación de la Junta de Andalucía.
} 
ratos públicos estatales, concebidos como agentes fundamentales para articular las relaciones sociales y promover el desarrollo. Con estas reformas se pretendía incrementar el intervencionismo del Estado, que durante esta etapa vio crecer de forma importante sus estructuras y dotaciones. Sin embargo, el balance global de estas iniciativas mostró escasos logros y, las reformas a gran escala, como esfuerzos sistemáticos y masivos destinados a transformar de forma substantiva la administración pública, no consiguieron implantarse ${ }^{2}$.

A partir de la década de los años ochenta aparecen nuevas iniciativas modernizadoras con un planteamiento diferenciado; en este sentido, se ha hablado de las denominadas «reformas de primera generación» que han supuesto (aunque la mayoría de ellas están todavía en marcha) la adopción de medidas que contribuyan a una reorganización del poder del Estado, y su consiguiente redistribución entre nuevos actores políticos y sociales. Entre ellas, aparecen por primera vez como prioridad en la agenda política, los llamados procesos de descentralización de poder hacia los Municipios ${ }^{3}$ y otras Entidades Locales, que sin duda alguna, son uno de los protagonistas de estos modelos. Su finalidad no era otra que racionalizar el ejercicio del poder público, mediante su distribución entre diversos actores, buscando el servicio al interés general de una manera más eficaz, eficiente, democrática y participativa.

Estas reformas se tradujeron, también, en la transformación del Estado en determinadas áreas de actuación (a través de privatizaciones, externalizaciones, y desregulaciones, entre otras), y en una importante reducción de efectivos de los aparatos públicos. Así, la vocación de estas iniciativas modernizadoras era de orientación externa del aparato estatal, es decir, pretendían modificar sus relaciones con la sociedad pero no su funcionamiento interno. Ello comportó que estas reformas se centrasen más en la reducción del servicio civil o función pública ${ }^{4}$ que en la transformación efectiva del modelo (más allá de introducir modificaciones como una mayor fragmentación y flexibilización).

Entrada la década de los años noventa surgió una nueva «generación» de reformas, que pueden diferenciarse netamente en cuanto a objetivos y orientación de la precedente. La distinción de estas «reformas de segunda generación» no debe ocultar ciertas líneas de continuidad con las anteriores, sin embargo, la preocupación de estas iniciativas reformadoras se situaba en la

\footnotetext{
${ }^{2}$ SPINK, P. (1997): «Technical Possibilities and Political Imperatives in 70 years of Administrative Reform». Paper prepared for the meeting of the Latin American Studies Association, Mexico.

${ }^{3}$ En torno al papel de los municipios puede consultarse el trabajo de CAstillo Blanco, F.; Zafra VícTOR, M.; Villalba PÉREZ, P. (2003): «El municipio en América Latina: reflexiones y propuestas». Revista de Estudios de la Administración Local, n² 291, pp. 251-281.

${ }^{4}$ La expresión «servicio civil», utilizada con preferencia en algunos países del área iberoamericana, se entenderá equivalente a la de «función pública».
} 
dimensión interna y, a modo de enlace con las reformas anteriores a los años ochenta, pretendían mejorar el funcionamiento de los aparatos administrativos públicos. En este segundo supuesto, se trataba de focalizar el punto de atención en la gestión eficiente de los recursos públicos redistribuidos entre los distintos actores políticos territoriales. Es decir, una vez realizadas las correspondientes transferencias de competencias a los Municipios, se trata ahora de adoptar las medidas concretas y necesarias que garanticen un ejercicio eficaz de las mismas.

Este objetivo se tradujo, entre otros, en el impulso de cambios en las reglas del juego de las relaciones entre el personal y la Administración, incidiendo en la propia conformación del modelo de servicio civil. Aunque todavía resultan vigentes, los resultados efectivos de esta segunda ola de reformas también se han cuestionado ${ }^{5}$, en especial por su incapacidad para transformar las instituciones vigentes en las distintas administraciones públicas.

Y en este contexto es, precisamente, el complejo tema del empleo público una de las cuestiones prioritarias a abordar dentro de estas últimas reformas. Garantizar la permanencia de las políticas públicas, profesionalizar la gestión, servir mejor a la ciudadanía, la lucha contra la corrupción ${ }^{6}$ y garantizar que los empleados públicos sirven a un Estado más democrático y a una Administración que, sometida al Derecho, tiene como fin el servicio al interés general son los principales objetivos de esta línea de actuación.

Es por ello que el estudio y análisis de los sistemas de función pública o servicio civil, adquiere una importancia notoria, en el marco de los procesos de modernización de las políticas públicas, y constituye un eje de las reformas de la gestión pública emprendidas por un número creciente de gobiernos en diferentes partes del mundo. Y es que, en el nivel local, la adecuada estructuración, profesionalización y motivación de los empleados públicos va a tener una incidencia decisiva en la efectividad del ejercicio competencial que se realice y en la consecución de los objetivos seguidos por las distintas políticas públicas.

No obstante, el servicio civil de carrera no es la solución a todos los problemas a los que se enfrentan las municipalidades, sino que necesariamente ha de verse como un factor instrumental que, junto a otros, puede ser determinante, especialmente determinante valga la redundancia, a los efectos de garantizar la permanencia y el éxito de las reformas del Estado que sean emprendidas.

\footnotetext{
5 Oslazk, Oscar (2001): «El Servicio Civil en América Latina y el Caribe: Situación y retos futuros», BID, Washington D.C.

${ }^{6}$ Véase sobre este tema, entre otras, la Convención de las Naciones Unidas contra la Corrupción (Resolución 58/4 del 31 de octubre de 2003) y la Convención Interamericana contra la Corrupción, aprobada por la Organización de los Estados Americanos el 29 de marzo de 1996, principales instrumentos jurídicos de carácter internacional referidos a la prevención y represión de actos irregulares cometidos por funcionarios públicos.
} 
Desde luego, ofrece ventajas difícilmente discutibles, entre otras, en los siguientes aspectos (lo que ha sido destacado por la mayoría de autores que se han ocupado del tema como Villoria, Prats, Longo, Merino, Méndez, etc...):

a) Reducción de la discrecionalidad irresponsable, asegurando, mediante el sometimiento al Derecho, la preeminencia del interés general y la objetividad en la acción pública.

b) Su contribución a reducir la corrupción dada la certidumbre jurídica laboral y su caracterización como un elemento que coadyuva a la transparencia en el quehacer gubernamental.

c) Fomento de la profesionalización, la dignificación del servicio público y la mejora en la implementación de las políticas públicas que, por este solo hecho, pueden programarse y ejecutarse en el tiempo sin ceñirse al período de gobierno.

d) Incentiva la rendición de cuentas, la supervisión, la evaluación.

e) Es un elemento básico para incrementar la confianza en el gobierno, la seguridad jurídica como base estratégica del desarrollo, la eficiencia asignativa, la durabilidad de los compromisos legislativos y un arreglo institucional garantizador de la eficiencia interna de los organismos y agencias administrativas.

f) Eliminación de la cultura de la lealtad individual por una cultura de la responsabilidad y la imparcialidad.

g) Genera seguridad laboral con base en el mérito y preserva la memoria institucional.

h) Permite la implementación de sistemas de capacitación permanentes y continuos diseñados desde el interés de la organización y no desde el interés del individuo, construir sistemas de incentivos vinculados a los objetivos estratégicos de la organización y, en general, gestionar los distintos subsistemas que conforman una gestión estratégica de recursos humanos.

Ciertamente, también puede presentar desventajas (uniformismo, rigidez, costes en su implementación, etc...), pero lo cierto es que, mérito y flexibilidad son, pues, elementos que ni se contraponen ni se disputan un territorio previamente limitado, como si se tratara de un juego de suma cero. Por el contrario, los nuevos diseños de servicio civil deben perseguir que ambas dimensiones se retroalimenten y desarrollen, en un círculo virtuoso de importancia crucial para la gobernabilidad de los sistemas político-administrativos contemporáneos ${ }^{7}$.

\footnotetext{
${ }^{7}$ LONGO, Francisco (2003): «La reforma del empleo público en las democracias avanzadas: mérito con flexibilidad» en Red de Gestión y Transparencia de la Política Pública, Servicio Civil: Temas para un diálogo, BID, Serie de Estudios Diálogo Regional de Política, Washington D.C.
} 
Y con independencia de dicha reflexión, es que, sobre todo en los países que carecen de servicio civil de carrera, si que existe una coincidencia en afirmar que las primeras superan con creces a los déficits citados en el párrafo anterior. Un dato confirma lo expuesto y es que, como ha puesto de relieve PRATS $^{8}$, la institucionalización de la función pública en su forma de sistema de mérito es un dato observable en todas las economías de mercado exitosas y en ninguna de las economías planificadas o de sustitución de importaciones. Y hay algo más y es que los valores de imparcialidad, mérito, lealtad al interés general, eficiencia y responsabilidad no son algo propio de los funcionarios, sino que son valores, necesarios para el desarrollo, que pertenecen a la sociedad. Uno de los actores, por ello, más interesados en que se construya un servicio civil de carrera ha de ser, por ello, la sociedad civil aunque en la mayoría de las ocasiones no sea totalmente consciente de ello.

\section{Los distintos modelos de servicio civil existentes en el ámbito comparado: sistemas abiertos vs. sistemas cerrados}

La evolución histórica que han experimentado los sistemas de servicio civil nos permite observar en los diferentes países un espectro muy variado de modelos y submodelos. Una variación que va desde los países que no poseen sistemas de servicio civil institucionalizados de base profesional; países que en la última década han intentado con mayor o menor éxito implantar de forma global o parcial un sistema de servicio civil; hasta países que poseen sistemas históricos de servicio civil, y actualmente están impulsado su revisión?.

A pesar de las enormes diferencias entre los distintos países, se pueden establecer algunos puntos de contacto entre los mismos ${ }^{10}$. Un primer rasgo característico se centra en la cuestión de la estabilidad del empleo público y su ordenación que se han vinculado con los dos grandes modelos de referencia en gestión de recursos humanos en las administraciones públicas. Se trata de la distinción entre un modelo cerrado que parte del principio de polivalen-

\footnotetext{
8 Prats i Catalè, J.(1995): «Los fundamentos institucionales del sistema de mérito: la obligada distinción entre función pública y empleo público». En Documentación Administrativa, 241-242; eneroagosto. Madrid: INAP.

${ }^{9}$ Sobre el estado de los servicios civiles en América latina puede consultarse los trabajos «Síntesis de los diagnósticos de servicio civil» en BID, Dialogo Regional de Políticas (2002) y «Estudio comparativo de estadísticas de empleo público en 26 países de América Latina y el Caribe» en BID (2003): «Red de Gestión y Transparencia de la Política Pública. Servicio Civil: Temas para un diálogo», Diálogo Regional de Políticas, Washington, D.C.

${ }^{10}$ LoNGo, experto en la materia de reconocido prestigio internacional, ofrece una clara visión comparativa de los distintos modelos en su obra «Mérito y flexibilidad. La gestión de las personas en las organizaciones del sector público». Ed. Paidós Empresa, 2004, Capítulo 2, pp. 78-86.
} 
cia de los empleados públicos (referidos como «funcionarios de carrera») y un modelo abierto inspirado en el principio de especialización ${ }^{11}$.

En la primera tradición, el Estado juega un papel central y muy visible como configurador de la sociedad. Como valores destacados de esta tradición se destacan los de seguridad jurídica, la equidad y la igualdad ante la ley. El modelo cerrado de servicio civil parte de la consideración de que el empleado público va a estar vinculado a lo lago de la vida profesional a la Administración, ocupando diferentes puestos de trabajo, y haciendo carrera a lo largo de ésta; uno de los primeros elementos distintivos de estas tradiciones administrativas es la estabilidad de sus sistemas políticos y administrativos. Con estas características los sistemas administrativos europeos de base continental (con referentes como Francia, Alemania o España y con las necesarias matizaciones en el último caso) representan los principios del modelo cerrado de servicio civil y adquieren un valor intrínseco como garantes de la continuidad, incrementando su autonomía e independencia respecto del sistema político.

En la segunda tradición, el papel del Estado es mucho menos significativo y visible, y tienden a limitarse y controlarse sus poderes, destacando el papel de los diferentes agentes sociales como configuradores de la sociedad. Los valores asociados a este modelo son los de la imparcialidad, la transparencia o el pragmatismo en la actuación de los gobiernos y administraciones. El modelo abierto de servicio civil, se basa en la diferenciación de los puestos de trabajo de la Administración, lo que supone hacer un estudio detallado de cada uno de ellos con el objetivo de reclutar a las personas más idóneas. El sistema administrativo anglosajón (y más específicamente los casos de Reino Unido y EE.UU.) es el que mejor refleja los criterios del modelo abierto de servicio civil en los que aparece una clara línea de continuidad, sin rupturas traumáticas o radicales, a diferencia de los modelos de la Europa continental en los que destaca la ruptura y transformación brusca del sistema político, mientras se mantiene estable el sistema administrativo.

Un segundo rasgo caracterizador de las dos tradiciones administrativas son las relaciones que se establecen entre la sociedad y los aparatos públicos. Los modelos anglosajones se desarrollan en un entorno que se acerca al «pluralismo», caracterizado por una sociedad civil muy dinámica, articulada y configurada de forma independiente de la intervención del Estado. Por contra, los modelos continentales tienden a configurarse en un contexto con una clara actividad intervensionista, o alternativamente en un contexto donde la articulación de grandes sectores de la sociedad condiciona la actividad del Estado, en una relación de apoyo mutuo.

\footnotetext{
11 Ballart, X.; Ramió, C. (2000), «Ciencia de la Administración», Tirant lo Blanch, Valencia; PaloMar, A. (2000), Derecho de la Función Pública. Régimen Jurídico de los funcionarios públicos. Dykinson, Madrid.
} 
En consecuencia con el planteamiento anterior, en el panorama internacional pueden observarse que las principales características que inspiran la construcción de los sistemas de empleo público abiertos son las siguientes:

- La idea clave de este sistema es la del puesto de trabajo que puede ser definido como la unidad estructural básica integrada por el conjunto de tareas y responsabilidades asignadas por la autoridad competente a una persona.

- Se basa en un inventario previo muy preciso de los puestos de trabajo, como se hace en las empresas privadas, limitándose la Administración a seleccionar las personas adecuadas que, con cierta frecuencia, provienen del sector privado.

- Se da preferencia en la selección a pruebas de tipo «práctico», entendiéndose por tales las que permitan determinar que aspirante puede hacerse cargo con más competencia del puesto de trabajo por el que se compite.

- La realización del reclutamiento para un puesto especial determina la exigencia de una experiencia previa en el desempeño de las funciones.

- Detrás de este sistema se asienta una Administración en línea con el sector privado, sometida al mismo ordenamiento que el resto de operadores jurídicos y que actúa muy descentralizadamente. La existencia de grandes empresas facilita el intercambio laboral entre el sector público y el privado.

- La Administración no tiene que ocuparse de la formación de los funcionarios, sistema de ascensos, situaciones administrativas, etc...

- La relación es generalmente de contrato laboral y los conflictos son resueltos por la justicia ordinaria.

- La flexibilidad y la rentabilidad son las notas características de este modelo.

- No existe la nota de permanencia, lo cual permite una gran flexibilidad al sistema, pudiendo adaptarse los efectivos de personal a las necesidades de los programas presupuestados. El personal que trabaja en la Administración es despedido cuando su puesto resulta innecesario o no es desempeñado con la competencia exigida.

Por el contrario, en la base del sistema de empleo público cerrado del modelo europeo continental-occidental sus características son diferentes:

- El elemento fundamental de este sistema es, en primer lugar, la posibilidad de contar con personal más cualificado, con una experiencia superior adquirida desde la iniciación al servicio, un perfeccionamiento 
adecuado a las funciones públicas y una continuidad asegurada en el desempeño de la función pública dada la permanencia.

- Las personas que ingresan en la Administración Pública, después de superar las diferentes pruebas de ingreso, entran en un cuerpo general o especial, normalmente jerarquizado, en el que pueden ascender desde los últimos niveles hasta los de más categoría. El ascenso se posibilita a través de la antigüedad, méritos o capacidad. Por regla general, los sistemas de promoción afectan, en forma negativa, a los poseedores de un cierto espíritu de empresa en favor de las personas capaces de trabajar en equipo y de manifestar un práctico espíritu de compromiso.

- El régimen jurídico de este sistema es el Derecho administrativo y no el Derecho laboral y se dan dos hechos fundamentales: la exigencia de un estatuto especial y la jerarquización.

- El comienzo en el servicio se produce en edades muy jóvenes, con exclusión de reglas excepcionales o de personas que, por méritos excepcionales, ingresan en la Administración Pública después de una experiencia profesional muy valiosa.

- Existe un sistema de requisitos individuales y de exámenes que en algunos países se completa con la realización de un curso en «Escuelas de funcionarios» y con un período de prácticas.

- Existe cierta incomunicabilidad entre los sectores privado y público.

- La rigidez del sistema de carrera obliga a la necesidad de establecer programas concretos de formación y adiestramiento, ya que la Administración debe utilizar sus medios personales, adaptándolos tanto a las meras actividades y servicios que presta como a las innovaciones tecnológicas que permitan la mejora en el desempeño de las funciones públicas tradicionales.

A la vista de los elementos definidores de cada uno de los modelos, se puede extraer una primera conclusión consistente en que la idoneidad de cada uno de ellos va a depender, en gran medida, del contexto, el sistema y la época en la cual hayan de hacerse valer. El papel de los sistemas de servicio civil en ambos casos se configura como una institución adaptada a contextos diferentes. Tanto la estabilidad del sistema político como las relaciones entre el Estado y la sociedad dan origen a sistemas administrativos diferenciados que, obviamente, facilitan el surgimiento y el mantenimiento de sistemas de gestión de los recursos humanos diferenciados. Mientras un modelo de servicio civil de carácter estable y continuado al servicio de un Estado fuertemente interventor se afianzaba en un contexto de inestabilidad política; en el polo opuesto, con un contexto políticamente estable y con una sociedad civil muy articulada, se conformaba un modelo de servicio civil de características diferentes al anterior. 
La discusión sobre su conveniencia tiende a centrarse sobre los valores que subyacen a ambos modelos, aunque ciertamente puede incurrirse en estereotipos. Se contrapone un modelo abierto asociado a la flexibilidad, al dinamismo y a la eficacia y la eficiencia, a un modelo cerrado vinculado a la rigidez y a la burocratización, que pueden tender a convertirse en un obstáculo para el funcionamiento de las organizaciones públicas.

Lo cierto es, sin embargo, que en una sociedad como la actual, con vertiginosos cambios en las tendencias de gestión de recursos humanos y con unas demandas cada vez más importantes de la ciudadanía hacia la Administración, un sistema rígido y cerrado con mayor dificultad puede dar respuesta a esas demandas y exigencias. Se necesitan instituciones flexibles cuya capacidad de adaptación al cambio sea posible. No obstante, los sistemas totalmente abiertos también ofrecen desventajas respecto de otros valores y principios que parecen relevantes y que es preciso reforzar en los países en desarrollo: la permanencia de las instituciones, los principios de ética pública ${ }^{12}$, la vocación de servicio de los empleados públicos, la independencia, imparcialidad, objetividad y trato igualitario en el trato con los ciudadanos... etc.

Todos estos extremos, y sus corolarios, son las consideraciones que habrán de valorarse a la hora de construir una carrera de servicio civil local en la que deberán ser extraídos los elementos más idóneos de uno y otro modelo, para poder cumplir así con los requerimientos de una Administración profesional y, en consecuencia, de una forma más eficaz y eficiente las demandas de nuestros ciudadanos.

\section{Balance de las experiencias internacionales: luces y sombras del impacto de la globalización de la gestión pública}

Como ya se aludió en un principio, los intentos de introducir sistemas de mérito en los modelos de servicio civil se remontan a la primera mitad del siglo $\mathrm{XX}$, aunque su concepción desvinculada de la estabilidad en el empleo y su falta de adecuación al contexto institucional en el que se aplicaba, dificultaron su institucionalización. En estos procesos destacó el papel jugado por los organismos internacionales, no siempre coordinados entre sí, cuya actuación incidió tanto en la elaboración de diagnósticos como en la definición de las medidas a emprender para su resolución. Las denominadas reformas de primera y segunda generación son claros ejemplos de la importancia de estos postulados, así como de la capacidad de influencia de determinados agentes.

\footnotetext{
${ }^{12}$ En torno a este punto es de sumo interés la consulta del informe «Iniciativas modelo en el ámbito del Estatuto de los funcionarios locales». Recomendaciones del Comité Director para la Democracia Local y Regional del Consejo de Europa. Serie Síntesis Ética Pública en el nivel local. Manual de Buenas Prácticas. Traducción y edición a cargo de José Manuel Rodríguez Álvarez, No 24, Octubre 2003, IUT- UIM.
} 
El hecho de que estas iniciativas se centrasen inicialmente en la mera reducción de las dimensiones del sector público y posteriormente tan sólo abordasen de forma parcial la transformación de ámbitos de gestión como el servicio civil se explica por los agentes involucrados en el proceso. Como indica BRESSER-PEREIRA, «estas reformas fueron dirigidas por economistas: los economistas locales y los economistas de las agencias internacionales como el Banco Mundial y el FMI (...), la mayoría de estos economistas son burócratas que tiene poca familiaridad con la gestión pública (...) por un lado tienen la idea de que un servicio profesional es algo bueno; por otro saben que los tiempos de la burocracia clásica terminaron (...). Así, tienden a dejar de lado la cuestión y a reducir la reforma del sector público al ajuste estructural, a la privatización, al downsizing y al combate a la corrupción» ${ }^{13}$. Pero los limitados resultados que ofrecieron, tan sólo destacables en cuanto a la reducción del volumen de empleo público, cuestionaron su validez como vía sustentable de actuación.

Entre las posibles causas, se apunta el hecho de que los cambios a los que se orientan las políticas y decisiones adoptadas no consiguen resolver plenamente el conflicto subyacente entre la racionalidad técnica en que se fundan y las condiciones culturales y políticas del medio en que pretenden ser implantadas. Por un lado, se constata la falta de voluntad por parte de los que forman parte de la estructura pública de incorporar nuevas técnicas que racionalicen las estructuras; y, de otro, se constata la subsistencia de un doble discurso que, aún dando el respaldo formal a las propuestas de reforma del servicio civil, continúan «sirviéndose» del mismo como fuente de poder. La brecha entre iniciativa y logro continúa siendo profunda ya que los fundamentos técnicos y axiológicos en que se basa el sistema de servicio civil o función pública profesional (mérito, responsabilidad, capacidad, equidad, transparencia...) son insuficientes para sobreponerse a los intereses, no sólo materiales, a que esas medidas afectan (privilegios, discrecionalidad, clientelismo, nepotismo, búsqueda de rentas, etc...), y que por consiguiente, no hacen más que poner de relieve la existencia de una cultura política clientelar y patrimonialista del empleo público ${ }^{14}$.

Con carácter general, el conjunto de experiencias internacionales en la materia ha supuesto que se haya llegado a un conjunto de reflexiones que es preciso atender para enfocar adecuadamente los procesos de construcción de servicios civiles de carrera.

Por de pronto, que estos procesos han de responder a la visión propia que cada país tiene del Estado, de su organización, de sus modos de actuación,

\footnotetext{
${ }^{13}$ Bresser-Pereira, L.C. (2001): «Reforma da nova Gestão Pública: Agora na Agenda da America Latina, no entanto», en International Journal of Political Studies, num. 3 (1).

${ }^{14}$ Para obtener una visión más completa sobre este punto puede consultarse el trabajo de PRATS (2000): «Del Clientelismo al Mérito en el Empleo Público. Análisis de un Cambio Institucional», Biblioteca $I D E A S$, Instituto Internacional de Gobernabilidad y Universidad Oberta de Catalunya.
} 
de su relación con la ciudadanía y de los cambios que se pretenden introducir, y entre estos últimos, la necesaria racionalización de la organización que exige, como paso previo, un diagnóstico de la situación que incluya los costes financieros que todo este proceso conlleva. En el caso concreto de las entidades locales, y si aceptamos los principios que rigen la nueva gobernanza, implica el replanteamiento y renovación de las formas de adecuación y organización de las administraciones públicas, consiguiendo unas organizaciones más flexibles y ágiles que no sólo garanticen la defensa de los derechos de los ciudadanos sino que, más allá de ellos, sean capaces de adoptar en forma proactiva la mejor decisión posible.

En segundo lugar que existe una coincidencia generalizada en que las reformas del empleo público integren, entre otros aspectos, una planificación flexible, una organización del trabajo en la que se concreten las tareas y perfiles de los puestos de trabajo, una gestión del empleo que permita una cierta movilidad, la racionalización de la política salarial, la existencia de sistemas de gestión del rendimiento que estimulen y retribuyan la contribución de las personas, y una adecuada gestión de las relaciones humanas y sociales en los que se desarrolla todo este entramado organizativo. Pero, precisamente, por esta razón la construcción de los servicios civiles de carrera en el nivel local ha de partir de una atenta lectura de las fortalezas institucionales que el nivel territorial local tiene en cada país para la puesta en marcha y gestión de ese sistema. Y, desde este punto de vista, la notoria ausencia de capacidad técnica y financiera de los Gobiernos locales resulta un primer punto a resaltar y del que se deben extraer consecuencias para la implementación exitosa del proceso.

En tercer lugar, que la construcción de servicios civiles de carrera no depende, simplemente, de la aprobación de un texto legal, sino que, más allá de ello, supone un conjunto de reformas institucionales (que incluye procesos culturales, pautas de conducta, normas no escritas, etc...) en las que la implementación de un texto legal es sólo un primer paso que exige ser enmarcado en una estrategia en la que se identifique las implicaciones que se derivan de la construcción de un servicio civil, con vocación de continuidad en el tiempo, y que responda a una estrategia liderada por el conjunto de instituciones involucradas y consensuada con los distintos actores políticos y sociales.

En esta misma línea, de forma más específica, las organizaciones internacionales han apuntado que el marco de prioridades en la gestión de los recursos humanos en el ámbito público, a los efectos de promover desde éste las políticas que coadyuvan al desarrollo son, al menos, las siguientes ${ }^{15}$ :

- La competencia en el mercado de trabajo, lo que implica el desarrollo de fórmulas nuevas que incrementen la capacidad de atracción de las

\footnotetext{
${ }^{15}$ OCDE. (2000a). «Summary Record of the Expert Meeting on Human Resources Management», 2526 January 2000. Paris: OECD.
} 
organizaciones públicas, en un contexto en el cual, como pone de manifiesto un documento sobre la situación en Holanda ${ }^{16}$, acrecentará al mismo tiempo el valor estratégico y la escasez de ciertas competencias clave.

- La promoción del liderazgo, en la que se centra el eje crucial del desarrollo de capacidad directiva en el sector público. Otro documento reciente explicita estrategias que pasan por la identificación del potencial, el desarrollo de la formación y el mentoring, la implicación de los gerentes en tareas de desarrollo de personas y la construcción de nuevos sistemas de incentivos ${ }^{17}$.

- La relación política/administración, que está en el núcleo mismo de los problemas de gobernabilidad de los sistemas públicos contemporáneos, en particular en lo que respecta a la consolidación de la esfera de dirección pública profesional.

- La gestión del conocimiento, que obliga a promover organizaciones públicas que aprenden, y a extender estos aprendizajes al conjunto del sector público. Todo ello exige políticas que incrementen el capital social interno de los sistemas públicos ${ }^{18}$, estimulando la formación de redes e intercambios basados en la confianza y la reciprocidad, y aprovechando el potencial de las tecnologías de la información y las comunicaciones.

- El fortalecimiento de la profesionalización pública, que apunta a la promoción de comportamientos éticos, al combate contra la corrupción y a la consolidación de los valores propios del servicio público en nuevos tipos de organización. En este sentido, la construcción de una infraestructura ética ${ }^{19}$, implica la actuación combinada en diversos frentes: el desarrollo legislativo, la transparencia y el impulso a una sociedad civil activa y vigilante, los sistemas de control interno y externo, los códigos de conducta y la enseñanza de la ética administrativa.

Por otra parte, llegados a este punto, se considera necesario poner de relieve algunas consideraciones acerca de las luces y también las sombras, que ha producido el impacto de la globalización de la gestión pública; en esta línea se constata, por un lado, impactos positivos entre los países desarrollados que poseen instituciones públicas sólidas y consolidadas; mientras que, por otro,

\footnotetext{
${ }^{16}$ OCDE. (2000b). «Comment trouver du personnel. Le cas de la fonction publique néerlandaise». Paris: OECD.

${ }^{17}$ OCDE. (2000c). «Developing Public Service Leaders for the Future». Background paper by the Secretariat. HRM Working Party Meeting. Paris: OECD.

${ }^{18}$ NAHAPIET, J., y GHoshaL, S. (1998): «Social capital, intellectual capital and the organizational advantage». En Academy of Management Review, vol.23, n 2, 242-266.

19 Villoria Mendieta, Manuel (2000): «Ética pública y corrupción: Curso de ética administrativa». Madrid: Tecnos.
} 
no se ha mostrado en absoluto positiva y funcional en aquellos países en desarrollo con instituciones marcadamente diferentes de las vigentes en los países de origen de las propuestas transformadoras.

Cabría preguntarnos, por tanto, ¿a qué se debe estos resultados tan difusos si el patrón seguido ha sido el mismo para unos y para otros? En este sentido, SERnA ${ }^{20}$, sostiene que el fenómeno de globalización de la gestión pública en los países en desarrollo ha supuesto de facto abandonar la vía institucional y optar exclusivamente por una concepción más tecnocrática. La revisión de los principales intentos de transformación muestra como la implantación de modelos de servicio civil no se hace mediante mecanismos institucionales, ya que no se considera el servicio civil como una institución, sino como un conjunto de instrumentos de carácter técnico. La raíz del problema radica, según el autor, en que no se han tenido en cuenta dos parámetros fundamentales:

a) Las instituciones son importantes y el servicio civil entendido como institución es relevante para la política de un país ya que su diseño juega un papel muy significativo en el proceso político. Las estructuras institucionales contribuyen a establecer las reglas del juego entre los distintos actores. Si no se considera al servicio civil como una institución los gobiernos tienden a alterar los elementos técnicos de un sistema de gestión de recursos humanos con el objetivo de modificarlo en base a intereses coyunturales y a las correlaciones de fuerzas existentes entre los actores administrativos.

b) El modelo más difundido de management público ${ }^{21}$ puede procurar eficacia y eficiencia en la provisión de bienes y servicios a ciudadanos individualizados. Esto es importante pero por sí mismo no genera un sistema de seguridad jurídica y de confianza, bases para construir la institucionalidad de un país. Es por ello que se argumenta que esta acepción del management público no genera suficiente valor añadido para generar instituciones públicas que aporten beneficios al sistema político y administrativo de un país.

Es por ello que no se puede sino concluir que no tiene sentido construir mecanismos de aprendizaje en la gestión pública en base a modelos de referencia globales cuando este proceso se hace sin un análisis de la realidad institucional previa. Por ello, es necesario destacar el papel de las instituciones en su aplicación concreta, en este caso en los sistemas de servicio civil, como punto de partida para el desarrollo de estrategias encaminadas a su transfor-

\footnotetext{
${ }^{20}$ Serna, Miquel S. (2003): «El impacto de los referentes internacionales en la transformación de las administraciones públicas latinoamericanas: agentes y dinámicas institucionales», VIII Congreso Internacional del CLAD sobre la Reforma del Estado y de la Administración Pública, Panamá, pp. 28-31.

${ }^{21}$ En torno a este punto puede consultarse el trabajo de EchEVERRíA, Koldo (2000): «La Administración Pública en la era del management», Monografías de Gobiernos Locales, CEMCI-UIM-ESADE, $\mathrm{n}^{\circ}$ 3, pp. 45-57.
} 
mación. Para ello se proponen cambios de carácter gradual basados en el reconocimiento de la propia realidad político-administrativa. Este enfoque parte de incorporar el aprendizaje mediante referentes internacionales, en un contexto de globalización, pero partiendo de la aceptación de las diferencias vinculadas a los contextos políticos, sociales y culturales de cada país, y proponiendo unas pautas de aprendizaje institucional y no exclusivamente tecnocrático. El aprendizaje global de los postulados de la gestión pública serán una vía adecuada para mejorar los sistemas administrativos, pero cuando se hayan conseguido rediseñar y consolidar instituciones sólidas que permitan aprovechar su potencial.

\section{PROPUESTAS PARA LA CONSTRUCCIÓN DE UNA FUN- CIÓN PÚBLICA LOCAL DE BASE PROFESIONAL EN AMÉRICA LATINA}

\section{Hacia la profesionalización de los servidores públicos en el ámbito local: variables de carácter esencial e instrumental}

A estos efectos, distinguiremos entre las variables que consideramos esenciales en el proceso de implementación de un servicio civil de carrera, de aquellas otras que, aún no teniendo este carácter, resultan de vital importancia para el éxito de una carrera de servicio civil municipal.

\subsection{Variables de carácter esencial}

A) Crear el marco institucional y el consenso político-social adecuado para la implementación de las reformas en la gestión de recursos humanos garantizando su permanencia en el tiempo.

El primer reto que se plantea es el de conseguir el liderazgo y un apoyo político suficiente y sostenido; de hecho liderazgo y apoyo político son necesarios porque los cambios cualitativos del management público implican casi siempre cambios en el equilibrio de influencias e intereses tejido en y alrededor de la organización concreta que se considere, en nuestro caso, el servicio civil de carrera ${ }^{22}$.

En efecto, es preciso definir qué se pretende, y definirlo con todos los actores implicados en el proceso, y una vez definido garantizar la permanencia de las políticas que aseguren su implementación. Pero, además, en el contexto

\footnotetext{
${ }^{22}$ Prats i CatalÀ, J. (2000): «El desarrollo del management público en la América Latina de los 90. La estrategia del M.P.D.» en «Pensar lo Público», Monografías de Gobiernos Locales, CEMCI-UIMESADE, n 3 , p. 160.
} 
local es preciso un diagnostico y estudio de la situación que tenga en cuenta las peculiaridades que este nivel territorial presenta.

El propio entorno político, económico y sociológico de las entidades locales en una era post-industrial, tecnológica, integrada en espacios geopolíticos altamente competitivos, cambiantes y de incertidumbre en que nos movemos en la actualidad, no es exactamente idéntico al que existía en el momento de creación de los servicios civiles de carrera tradicionalmente puestos como ejemplo. Asimismo, la exigencia de resultados dista mucho de ser la que la ciudadanía exigía al comienzo de los procesos de descentralización $^{23}$. Por último, la madurez y el desarrollo organizacional de los Gobiernos locales tras estos procesos, sin perjuicio de que subsistan carencias relevantes, tampoco es igual, ni asimismo el grado de desarrollo competencial de las mismas que en su ejercicio manifiestan una diversidad notoriamente más acusada que otras Administraciones territoriales.

Pero, asimismo, también es preciso referirse a un elemento que ha de ser abordado específicamente en el nivel local. Y es que, efectivamente, un conjunto de elementos condicionan el modelo de organización territorial local y ponen en jaque una visión estática de la organización del territorio, a saber: una realidad municipal heterogénea en la que conviven municipios con una dimensión que no le permite gestionar con eficiencia las competencias a ellos atribuidos y, junto a éstos, municipios de gran tamaño que gestionarían con mayor eficacia competencias atribuidas a otras administraciones públicas; aceptación de la existencia de entes territoriales de idéntico nivel, pero con una necesidad de prestación de servicios distinta determinada por la existencia de factores específicos que los singularizan (municipios turísticos, de montaña, metropolitanos, etc...); la existencia de realidades urbanas integradas por varios términos municipales y que crean una problemática urbana común; las infraestructuras de transporte terrestre que facilitan la movilidad y la residencia en lugares distintos; las dinámicas ambientales derivadas de la existencia de espacios naturales y que exigen un tratamiento conjunto más allá de los límites municipales o departamentales; las nuevas necesidades que la sociedad en materia de ocio y consumo plantea; o, por poner un último ejemplo, las necesidades asimétricas en relación a su dimensión geográfica y humana que exigen la prestación de ciertos servicios públicos (agua, residuos, transportes, etc...). Todo ello, aparentemente ajeno al empleo público, no lo es. Y las adecuadas soluciones que hayan de encontrarse habrán de tener en cuenta esta realidad.

B) Fortalecer la dirección de recursos humanos de las municipalidades e incrementar la apuesta por la formación como un factor estratégico para el desarrollo de la organización y de sus recursos humanos.

\footnotetext{
${ }^{23}$ Véase el trabajo de Montesinos, Egon (2005): «Los estudios de descentralización en América Latina: una revisión sobre el estado actual de la temática». Revista EURE (Vol. XXXI. No 93), pág. 77-88, Santiago de Chile.
} 
La observación de la realidad actual en el campo público en la mayoría de los países donde se ha implementado un servicio civil de carrera pone de relieve que las funciones de estas unidades refleja, con carácter general, un panorama no satisfactorio si atendemos a las funciones desarrolladas por las mismas. Situación que, además, resulta acentuada en el caso de los Gobiernos locales.

En efecto, la gestión de los recursos humanos se realiza sin establecimiento de objetivos ni plan alguno, sin evaluación de los resultados, sin una clara definición del contenido y la dimensión de las unidades orgánicas, en medio de la ausencia de una política de recompensa/sanción, con un importante déficit en los mecanismos de racionalización y reasignación de efectivos en la que, en ocasiones, se opera con una simple lógica incrementalista, con una utilización de la formación en la que sólo se tienen en cuenta los intereses de desarrollo personal de los miembros de la organización, etc...

Es preciso que la Dirección de Recursos Humanos, sin embargo, no simplemente aplique la norma que eventualmente se dicte, sino que la dirección ha de plantear el problema, valorar las posibles soluciones en términos de recursos, priorizarlas en función de las posibilidades existentes, etc... En definitiva, la función de recursos humanos ha de considerarse como una variable estratégica de la organización que ha de incorporar nuevas habilidades acordes con dicha concepción.

Desde este punto de vista, también ha de considerarse que los sistemas de gestión de personas no son un patrimonio exclusivo de las unidades de recursos humanos, sino que son una realidad transversal y estratégica que alcanza a la organización como las políticas de calidad o de financiación. Por ello es preciso la desconcentración de numerosas parcelas que habitualmente se desarrollan en forma centralizada en las unidades de recursos humanos, centrando las mismas en la definición estratégica de dicha gestión y no en la administración del estatuto en la que deben implicarse, asimismo, el resto de unidades de la organización.

Por otra parte, desde la perspectiva de las administraciones públicas, la formación profesional continua y el reciclaje profesional de todos los empleados públicos ha constituido desde siempre una necesidad imprescindible en orden a conseguir sus objetivos permanentes de eficacia y de modernización. Pero, acaso hoy en día, su necesidad sea aún más sentida, dada la rápida evolución de los conocimientos y de las tecnologías de la información y de las comunicaciones y, con ello, de los requerimientos de la Administración a sus empleados. La formación constituye un factor relevante para incrementar la productividad de cada empleado público y de la organización en su conjunto. Pero es que, además, la formación no sólo contribuye a la mejor calidad del trabajo desarrollado, sino que sirve como instrumento de motivación y compromiso del personal, de transmisión de cultura y valores, de progreso personal y profesional, y de transmisión y conservación del conocimiento. Todos estos ele- 
mentos influyen de modo directo en el mejor desempeño y son uno de los signos distintivos de las organizaciones más avanzadas y eficientes. No por ser un tópico deja de ser verdad la afirmación de que la formación es una inversión, una de las mejores inversiones que puede hacer cualquier organización.

Paralelamente, desde la posición de los empleados públicos, la formación profesional constituye sin duda el prius lógico para poder llevar a efecto una carrera profesional, promocional o retributiva que debe ser reconocida como un derecho y como un deber de éstos.

Desde este punto de vista, los Gobiernos locales han de adoptar, de esta manera, un modelo «positivo» o activo de formación profesional, en virtud del cual deben asumir, por si mismos o en forma coordinada y apoyados por la cooperación internacional, tanto las cargas que requiere proporcionar la formación profesional necesaria o conveniente para sus empleados, como el reto de optimizar la utilización de los recursos destinados a estos fines.

C) Sensibilizar a los actores con incidencia y socializar las propuestas: el proceso de implementación del servicio civil de carrera es un proceso cultural además de normativo.

En efecto, por encima de las anteriores reflexiones, es preciso realizar una de mayor calado y que puede explicar, en gran medida, la persistencia y prácticamente nula desaparición de los problemas que han venido apareciendo, con una lógica implacable, tras la aprobación de distintos instrumentos legales reguladores del empleo público en diversos países ${ }^{24}$.

La clave reside en admitir con humildad y modestia que no basta un cambio normativo para producir, a modo de ungüento milagroso, un cambio en el sistema establecido. Efectivamente, PrATs ha puesto de relieve la necesidad urgente, para que las reformas del Estado alcancen el éxito que se espera de las mismas, de que se produzca un cambio institucional. Quiere decir ello, en palabras de dicho autor, que «las organizaciones estatales no son libremente disponibles; contrariamente, se encuentran ampliamente sobredeterminadas por el tipo de institucionalidad pública existente, es decir, por las normas y reglas -formales e informales - que establecen los fines, límites, actores, procedimientos y formas de participación en la acción colectiva, así como por los correspondientes modelos mentales, actitudinales y valorativos que prestan legitimidad, permiten la vigencia y dificultan el cambio de tales reglas y normas» ${ }^{25}$. De todo lo cual deduce el autor una conclusión: «si la institucionali-

\footnotetext{
${ }^{24}$ Véase como modelo de instrumento legal la Carta Iberoamericana de la Función Pública aprobada en el marco de la XIII Cumbre Iberoamericana de Jefes de Estado y de Gobierno en Santa Cruz de la Sierra, Bolivia, Noviembre 2003.

25 Prats i Català, J., (1997): «Fundamentos conceptuales para la reforma del Estado: el redescubrimiento de las instituciones» en La reforma de los legislativos en América Latina, Tirant lo Blanc, Valencia y «Reforma del Estado y desarrollo humano en América Latina», Revista Quórum, Universidad de Alcalá de Henares, Madrid, nº 1, p. 44.
} 
dad pública es el marco de incentivos de las organizaciones públicas, cualquier reforma de éstas sólo será positiva y duradera en la medida en que contribuya a reformar también positiva y duraderamente el fondo o marco institucional en que la organización se inserta» y, correlato de lo anterior, que si reconocemos como cierto lo expuesto la reforma del sistema de empleo público no puede ser solamente una operación meramente técnica sometiéndola a una lógica de cambio perfectamente planificado.

Mutatis mutandis, y aplicada dicha reflexión al objeto de nuestro análisis, difícilmente variaremos un ápice el sistema de empleo público mientras no modifiquemos los valores y marcos en que se inserta el mismo. Podremos variar su estructura y composición, profesionalizar a los empleados públicos, potenciar el sistema de carrera, introducir la negociación colectiva, flexibilizar la relación jurídica entablada, dinamizar el sistema retributivo, etc..., pero lo que a la larga verdaderamente importa permanecerá invariable y es que los sistemas de empleo público también obedecen a patrones de comportamiento, a modelos, a lógicas y tabúes que de no modificarse determinarán que unos cuantos años después de establecido un nuevo sistema volvamos a reiterar las mismas dificultades y los mismos viejos problemas aunque formalmente se respeten las normas.

D) Diseñar un modelo de implementación de un sistema integral en el que se fortalezca la profesionalización del personal al servicio de los Gobiernos locales.

La implementación de un servicio civil de carrera, y la consiguiente profesionalización del personal al servicio de los Gobiernos locales, debe servir, asimismo, como una estrategia dirigida a afrontar los eventuales problemas de corrupción y ausencia de transparencia que presenten los Gobiernos locales y estar orientado, en última instancia, a la mejora de la prestación de los servicios a los ciudadanos.

La construcción de este servicio civil tiene que tener como horizonte una redefinición de las organizaciones en que todo el personal al servicio de éstas tenga una posición activa, con capacidad de fijación de metas, autorrealización y aceptación de responsabilidades. Esto implica gestionar los recursos humanos con modelos de alto compromiso basados en los cuatro principios enumerados por LAWLER: 1) Información sobre los procesos, los estándares de calidad, opinión de los clientes, funcionamiento de la organización y de los resultados; 2) Conocimientos, en el sentido de formación, sobre su trabajo, los objetivos y el sistema global de la organización; 3) Poder, es decir, delegación para tomar decisiones en lo que concierte a su trabajo; 4) Recompensas, ligadas a los resultados y crecimiento en la contribución y en las capacidades del factor humano ${ }^{26}$.

\footnotetext{
${ }^{26}$ LAwLER, E. (1995): «Creating High perfomance organizations», Jossey Bass Publishers, III.
} 


\subsection{Variables de carácter instrumental}

A) Facilitar la flexibilidad y la cooperación organizativa haciendo compatible la especialización con la versatilidad de las funciones atribuidas a los puestos de trabajo y articular sistemas que hagan viable la movilidad interna y externa entre las distintas funciones públicas de los entes locales y el Estado.

Una de las desventajas que presentan los servicios civiles de carrera, ya se ha dicho, consiste precisamente en su eventual rigidez. Precisamente por ello el diseño del mismo debe responder a las funciones y competencias del Gobierno local, a su organización institucional y a los servicios que efectivamente se prestan. Y, además, hacerlo de forma flexible y adaptativa atendiendo a necesidades futuras y cambios en las formas de gestión y actuación del nivel local.

B) Articular un sistema salarial sensible y sencillo que potencie las competencias y el desempeño.

Las retribuciones del personal al servicio de los Gobiernos locales alcanza en la mayoría de las municipalidades de los distintos países una muy importante partida de sus presupuestos y ello exige, aunque sólo fuese por la importancia económica que suponen en el total de recursos disponibles, la necesidad de que el sistema retributivo se canalice en forma eficiente para estimular las actitudes y comportamientos de las personas y, de esta manera, poder contribuir al cumplimiento de los objetivos de la organización.

Desde este punto de vista, y con independencia del tratamiento que se le dé en la norma, el objetivo ha de ser que la cuantía del salario y su administración sea adecuada y con visión estratégica. Si el sistema retributivo no responde a una estrategia y a unos objetivos claros pronto perecerá en su coherencia interna debido a las presiones corporativas de grupos de empleados o al deseo de satisfacción de personas singulares dentro de la organización.

En cualquier caso, debe advertirse que dos de los más importantes problemas que deberán enfrentarse en este aspecto consisten: el primero de ellos, en la insuficiente cuantía del salario que puede determinar unas organizaciones poco productivas; el segundo, y no menos relevante, en un importante grupo de países, no es tanto el montante retributivo, sino más bien la desigualdad en la aplicación de éste entre los empleados públicos ${ }^{27}$.

C) Conocer la situación de gestión del empleo público local y su contexto: una nueva visión y un nuevo ámbito de las relaciones laborales en el sector público local.

\footnotetext{
27 Sobre este tema puede consultarse el trabajo «La Remuneración de los Altos Dirigentes del Sector Público Un análisis sobre los países de América Latina» elaborado por Nelson MARCONI, LAURA CARRILlo y Claudia Helena CAVAlieri, bajo la coordinación del CLAD.y comisionado por el Banco Interamericano de Desarrollo para el Diálogo Regional de Política, diciembre de 2003.
} 
La relación antagónica y de desconfianza eventualmente existente entre la representación sindical y el Gobierno local ha de ser superada y establecida una nueva cultura de cooperación y colaboración en la mejora de la calidad de vida laboral de los empleados al servicio de los Gobiernos locales. Esto requiere reconstruir el Gobierno local con los trabajadores y en ningún caso para, contra o sin la participación activa de éstos y el desarrollo de una estrategia de formación de los actores y responsables de las relaciones laborales en formas alternativas de solución del conflicto y en enfoques conjuntos de resolución de problemas.

Del mismo modo es preciso diseñar un procedimiento de participación de los empleados públicos en el establecimiento de sus condiciones de trabajo que ha de tener en cuenta: las restricciones propias en cuanto al ámbito de negociación al tratarse del sector público; la estructura municipal de cada país; la falta de confianza en un sistema de relaciones laborales y en el papel de los sindicatos.

D) Articular un sistema reaccional y de garantía jurisdiccional como mecanismo de cierre del sistema y creación de mecanismos arbitrales de solución de conflictos.

A nadie se le oculta la alta conflictividad, tanto en las relaciones laborales como en las funcionariales en el caso de los países que tienen implementados servicios civiles de carrera. Sin embargo, dicho estado de cosas no es satisfactorio ni para los trabajadores que aprecian abusos manifiestos, ni para los tribunales que se ven abocados a resolver imperativamente conflictos que podrían tener otras vías de solución, pero ni, inclusive, para las propias organizaciones públicas cuya actuación en este concreto aspecto no potencia la motivación de los empleados públicos y, en ocasiones, ofrecen una sensación de abuso injustificable en la toma de decisiones, articulada de forma unilateral en la mayoría de los supuestos, que no favorece la armonía en las relaciones laborales.

\section{Los desafíos pendientes para alcanzar el éxito de las reformas emprendidas}

A) El contexto interno y externo como factores determinantes de la definición de un sistema de servicio civil: falta racionalizar la organización y un diagnóstico integral.

La eficacia de los principios, procedimientos, políticas y prácticas de gestión que configuran un sistema de servicio civil requiere que todos ellos sean debidamente contextualizados en el entorno institucional en el que deben incardinarse y operar. Los requerimientos derivados de la historia, las tradiciones, el contexto socioeconómico y el marco político de cada realidad nacional son factores que condicionan los contornos específicos de cualquier modelo genérico. 
En este contexto, la gestión de RRHH que no se desarrolle, con arreglo al anterior parámetro, dará lugar a una visión muy restringida, ausente de planificación alguna, y sin gestión de la mayoría de subsistemas que integran la función de RRHH.

Asimismo, se constata la ausencia de un diagnóstico de la situación, y por ende, una falta de información que permita conocer los desafíos pendientes que son necesarios abordar, como paso previo, a la hora de afrontar el reto de construir una carrera de servicio civil municipal.

B) Existencia de una cultura política clientelar, falta de capacidades para la construcción de un servicio civil y resistencia al cambio: el diseño institucional político y la competencia electoral.

La génesis de la problemática de recursos humanos en los municipios parece responder a una posible poco evolucionada capacidad política de sus cargos electos y a una cultura popular que es permisiva con prácticas clientelares y de favoritismo político. La burocracia pública en las municipalidades no está al servicio objetivo e imparcial del interés general, sino al servicio de aquél que, en ese momento, desempeña la Alcaldía de la localidad.

En consecuencia, el pluripartidismo, la debilidad de los partidos políticos, la fragmentación de los actores públicos, la inestabilidad política, la existencia de una Asociación de Municipalidades con capacidades de gestión e interlocución, la competencia electoral, son factores todos ellos (y sus contrarios), los que pueden contribuir al éxito o fracaso de las reformas emprendidas ${ }^{28}$.

C) Deficiencias en la capacidad institucional de las municipalidades: falta de capacidades para la gestión del sistema.

Una característica común que se pone de relieve de forma reiterada, consiste en la constatación de una deficiente capacidad de gestión de un sistema de carrera administrativa por parte de la mayoría de las municipalidades. Las principales causas de esa incapacidad son básicamente dos:

- Insuficiencia financiera de la entidad local para hacer frente a las medidas que conlleva la implantación de un sistema de carrera profesional.

- Insuficiencia de equipos técnicos cualificados para la construcción de una carrera administrativa municipal.

Estos factores negativos van asociados a una visión de la autonomía local todavía embrionaria y apegada a esquemas paternalistas de supervisión

\footnotetext{
${ }^{28}$ Para profundizar sobre la cuestión política en las reformas del servicio civil véanse los trabajos de Heredia, Blanca (2002): «Reforma del Servicio Civil en América Latina: Atando las manos de los políticos», y «La economía política de la creación de servicios civiles de carrera: La experiencia de México en los años 90», CIDE, BID, México.
} 
y control del Estado. Ese escaso desarrollo de la institucionalidad política local, se entiende si se tienen en cuenta que sólo recientemente ha sido realmente dotada de reconocimiento jurídico la autonomía local al permitir la elección por sufragio universal de los alcaldes y el resto de regidores que conforman la corporación municipal y desechar la costumbre de nombramiento por parte del poder ejecutivo estatal de dichos cargos políticos locales.

Existe, con independencia de lo anterior, un déficit de capacidad en numerosas municipalidades que es preciso suplir, a través de fórmulas asociativas y con la creación de un órgano ad hoc que coadyuve en dicha tarea.

D) Crisis fiscal: la construcción de un servicio civil genera costes que las municipalidades no podrían financiar.

Una de las principales rémoras de gestión de los recursos humanos se centra en la escasa autonomía de los municipios, sobre todo en lo referido a su capacidad recaudatoria y presupuestaria. En consecuencia, se reconocen muy mermados en cuanto a posibilidades de implantar reformas o medidas de fortalecimiento de sus estructuras básicas y de su organización interna.

En efecto, la implementación de un servicio civil de carrera implica costes. Costes por su eventual repercusión sobre el sistema salarial existente, por su impacto sobre el sistema de clases pasivas y por las necesidades derivadas de su implementación.

E) Falta de conciencia social y de los beneficios que genera su implementación: las reticencias culturales a la puesta en marcha de cambios en los sistemas de gestión del empleo público.

Es opinión generalizada lo beneficioso ${ }^{29}$ de contar con unos recursos humanos estables, profesionales y capacitados para desarrollar las labores administrativas y de desarrollo económico de los municipios. Partiendo de esta premisa, podemos decir, sin embargo, que la viabilidad y oportunidad de la reforma no es asumida con igual entusiasmo por todos.

Las prioridades de la agenda política no dotan de protagonismo a los componentes municipales de las reformas, lo que da una impresión de que la reforma del empleo estatal y el municipal son consideradas dos líneas de trabajo paralelas. Así, se constata la dinámica por parte de numerosos gobiernos nacionales de excluir a los empleados municipales.

Al mismo tiempo, es arraigada costumbre el que los empleados municipales sean removidos de sus puestos cuando cambia el líder local, aunque casi

\footnotetext{
${ }^{29}$ Diversos estudios realizados por diversos organismos internacionales como el BID, el CLAD o la CEPAL ponen de manifiesto una correlación directa entre las capacidades del Estado y el desarrollo de los países, así como una relación positiva entre la existencia de sistemas de función pública o servicio civil eficientes y los niveles de confianza de los ciudadanos en la Administración Pública, la eficiencia gubernamental y el crecimiento económico sustentable de los países.
} 
todos los implicados rechazan estas prácticas por ser de elevado coste y un factor evidente de ineficacia para la prestación de los servicios públicos municipales.

En este sentido, es palpable una desconfianza hacia la capacidad de las instituciones públicas para afrontar el reto de la profesionalización del empleo público y una opinión poco positiva acerca de la voluntad política de las instancias nacionales y de los alcaldes de liderar la reforma que aquí se propone. No se tiene esperanza en un decidido apoyo desde instancias supralocales y se confía en que la tarea de modernización administrativa y del empleo público municipal es algo que debe ser liderado y protagonizado por sus propios cargos públicos mediante la unión de sus fuerzas.

Esta situación pone de manifiesto una ventaja y una debilidad; por un lado, existe conciencia de la necesidad de adoptar cambios relevantes en el tratamiento del empleo público local; pero, por el otro, se evidencia una desconfianza hacia la capacidad de realizar ese cambio.

F) Desorden en la actuación de la cooperación internacional.

Como ya se apuntó al inicio del presente artículo, los resultados del impacto de la globalización de la gestión pública no han sido del todo positivos, sino más bien, podrían considerarse difusos.

Y es que, en efecto, se ha observado cierto desorden en la actuación de los organismos internacionales, ya sea, bien por la ausencia de sinergia positiva (oferta), bien por la falta de estructura en la demanda, o bien por la no priorización en las necesidades, lo cierto es, que no se ha producido una coordinación de acciones, ni mecanismos que las coordinen, a la hora de desarrollar estrategias encaminadas a transformar los sistemas de servicio civil ${ }^{30}$.

Es por ello que estas prácticas no hacen más que evidenciar la necesidad de mecanismos de coordinación de acciones que favorezcan la permanencia en el tiempo de las reformas que pretendan ser implementadas.

En todo caso, lo que se determina es la importancia de construir un proyecto de servicio civil municipal ${ }^{31}$ que escuche a todas las partes implicadas y que saque de la desidia y la inoperancia al funcionamiento de los municipios. Para ello se ve como imprescindible el convencimiento intimo de todos los afectados de los beneficios que a largo plazo podrán disfrutarse y el compromiso cierto de los políticos para que destinen esfuerzo personal y partidas económicas suficientes para su implementación.

\footnotetext{
${ }^{30}$ Una acertada reflexión entorno a la transformación de los sistemas civiles se encuentra en GUERRA P., Alberto (2003): «Antecedentes, realidades y perspectivas de la carrera administrativa», VII Congreso Internacional del CLAD sobre Reforma del Estado y de la Administración Pública, Panamá..

31 Sobre la implantación de una carrera de servicio civil municipal puede consultarse el trabajo de Martínez Rosiles, Jaime (2005): La profesionalización y el Servicio Civil de Carrera y su implantación en las Administraciones Municipales, Michoacán, Universidad Latina de América.
} 